\title{
Intervención en drogas desde atención primaria: revisión de guías clínicas
}

\author{
Verónica Casado Vicente*; Daniel Araúzo Palacios** \\ * Médico de familia. Presidente de la Sociedad Castellana y Leonesa de Medicina de Familia y Comunitaria. \\ * Médico de familia. Vocal de Grupos de Trabajo de la Sociedad Castellana y Leonesa de Medicina de Familia y Comunitaria. \\ Enviar correspondencia a: \\ Verónica Casado Vicente. Centro de Salud Parquesol. C/ Ciudad de la Habana 7. 47014 Valladolid. Tel. 983-380221. vcasado@teleline.es
}

Recibido: 31 de marzo de 2003. Aceptado: 24 de abril de 2004.

\section{RESUMEN}

Objetivo: Identificar las intervenciones sobre las conductas de riesgo adictivo, basadas en la evidencia, susceptibles de desarrollo en Atención Primaria.

Diseño: Revisión sistemática de programas y guías de práctica clínica basada en la evidencia.

Fuente de datos: Bases de datos Medline.

Selección de estudios: Recomendaciones PAPPS, US Preventive Services Task Force, Canadian Preventive Task Force, American Medical Association (AMA), AMA Guidelines for Adolescent Preventive Services (GAPS), American Academy of Family Physicians (AAFP) y Veterans Health Administration.

Extracción de datos: Se listan las actividades de intervención en drogas y se comprueba en las guías seleccionadas su nivel de evidencia y recomendación, observándose las concordancias y discrepancias.

Resultados: El abordaje en las conductas adictivas debe realizarse en todo el sistema sanitario, siendo la coordinación entre niveles sanitarios y los recursos comunitarios la clave del éxito. Los recursos normalizados, en concreto la Atención Primaria, son determinantes para las actividades preventivas: detección precoz, detección de situaciones y conductas de riesgo, reducción de riesgos, abordaje familiar, inicio de algunas desintoxicaciones y los cuidados paliativos, con apoyo externo si es necesario. El fomento de estilos de vida debe basarse en la coordinación con maestros y padres. Y la deshabituación y reinserción social con los recursos de segundo nivel. La formación de los profesionales y la mejora de la calidad de las evidencias científicas, así como la medición de los impactos propios de las actividades a nivel de la comunidad, son también claves en Atención Primaria.

Conclusiones: Existen evidencias sólidas sobre diversas actividades a realizar en la intervención en drogas desde Atención Primaria.

Palabras clave: Conductas adictivas, Abuso de drogas, Manejo de Drogodependencia, Atención Primaria, Medicina de Familia.

\section{ABSTRACT}

Objectives: To identify the interventions in high-risk addictive behaviours, based on the evidence, with the potential for development in primary health care.

Design: Systematic review of programmes and clinical guidelines based on the evidence.

Data source: Medline database

Bibliographic sources: PAPPS recommendations, US Preventive Services Task Force, Canadian Preventive Task Force, American Medical Association (AMA), AMA Guidelines for Adolescent Preventive Services (GAPS), American Academy of Family Physicians (AAFP) and Veterans Health Administration.

Data extraction methods: We listed drug intervention activities and checked their levels of evidence and grades of recommendations in the selected guidelines, observing concordances and discrepancies.

Results: An approach to addictive behaviours must be made throughout the entire health system, the key to success being coordination between all health care levels and community resources. Standardised resources, specifically primary care, are determinants in preventive activities: early detection, detection of risk situations and behaviours, harm reduction, the family approach, initiation of some drug rehabilitation and palliative cures, with external support where necessary. Promoting alternative life styles must be based on coordination between teachers and parents, and giving up drugs and social reinsertion with secondary level resources. Training professionals and improving the quality of scientific evidence as well as measuring the impacts of the actions at a community level, are also keys, particularly in primary health care.

Conclusions: Solid evidence exists on diverse activities to be carried out in interventions in drug dependency in primary health care.

Key words: Addictive behaviour, Drug abuse, Management of substance use disorders, Primary health care, Family Medicine. 


\section{INTRODUCCIÓN}

$\mathbf{L}$ as características esenciales de la Atención Primaria, como la accesibilidad, la globalidad y la continuidad de la atención, la confieren especial relevancia y la colocan en una situación privilegiada para la atención a las personas como un todo en su entorno familiar y social. Estas características posibilitan atender a las personas en las diferentes etapas de su vida con el objetivo de elevar el nivel de salud: favoreciendo estilos de vida, detectando situaciones de riesgo, disminuyendo los riesgos, curando, rehabilitando y paliando.

En el amplio abanico de servicios que la Atención Primaria y el Médico de Familia debe ofertar en cuanto a atención al individuo, atención a grupos poblacionales y de riesgo, atención a la familia y a la comunidad, se encuentra la atención a las conductas de riesgo adictivo.

La extensión de estas conductas, su gravedad, su vulnerabilidad, su evolución en el tiempo y su repercusión social convierten su atención en una actividad prioritaria y en una actividad a incluir en las carteras de servicios de los centros de salud.

Ahora bien, el abanico de actuación es muy amplio. Las actividades a ofertar en este campo se apoyan en diversos bloques de actividad 1,2,3.

Diferentes organismos nacionales e internacionales se pronuncian sobre las actividades a realizar y algunos, a la luz de las evidencias, proponen recomendaciones. El objetivo de este estudio es precisamente identificar las intervenciones que han demostrado un efecto en la disminución de la morbimortalidad asociada a las conductas de riesgo adictivo y promover el manejo de pacientes con conductas de riesgo adictivo, fundamentado en actividades basadas en la evidencia. Se trata de identificar las actividades a realizar y sus diferentes grados de evidencia y recomendación y, a la luz de todas las evidencias, hacer una propuesta concreta de cartera de servicios para Atención Primaria.

\section{MATERIALY MÉTODOS}

Se trata de una revisión normalizada de acuerdo con las herramientas elaboradas por los distintos grupos de Medicina Basada en la Evidencia ${ }^{4-13}$. Se ha efectuado una búsqueda de programas y guías de práctica clínica basadas en la evidencia en las bases de datos Medline. Las guías que se seleccionan se han desarrollado sobre búsquedas del registro de ensayos clínicos controlados de Cochrane y sus conclusiones se desprenden, preferentemente, del análisis de metaanálisis, ensayos clínicos controlados y randomizados o estudios casos control, y se seleccionan preferentemente las que aúnan la experiencia clínica en prácticas basadas en la evidencia con las mejores evidencias clínicas disponibles derivadas de la revisión sistemática.

Se han utilizado como descriptores los siguientes términos: conductas adictivas, abuso de drogas, manejo de drogodependencia, atención primaria y medicina de familia. Se han excluido todas las guías y programas cuyas conclusiones no se basaron en las evidencias científicas o las que no tenían en cuenta la experiencia clínica. Se han listado las actividades de prevención primaria, secundaria y terciaria en las que debería intervenir la Atención Primaria, sóla y en coordinación con otros niveles y sectores, según los textos de uso habitual en Atención Primaria y programas sobre drogodependencias ${ }^{1,2,3}$. Se han revisado las recomendaciones del Programa de Actividades Preventivas y de Promoción de la Salud (PAPPS) ${ }^{4}$, de la US Preventive Services Task Force 5,6,7,8, de la Canadian Preventive Task Force $^{9}$, de la American Medical Association (AMA) ${ }^{10} \mathrm{y}$ la AMA Guidelines for Adolescent Preventive Services (GAPS) ${ }^{11}$, de la American Academy of Family Physicians $(A A F P)^{12}$ y de la "Veterans Health Administration" ("The clinical practice guideline for the management of substance use disorders" $)^{13}$.

La clasificación empleada para los niveles de evidencia y la fuerza de la recomendación se expone en la tabla I, según el esquema de la US Preventive Task

\section{TABLA I. NIVELES DE EVIDENCIA Y FUERZA DE LA RECOMENDACIÓN}

\section{CALIDAD DE LA EVIDENCIA}

I: Evidencia obtenida al menos de un ensayo clínico controlado aleatorizado de manera apropiada

II-1: Evidencia obtenida de ensayos clínicos controlados bien diseñados sin aleatorización

II-2: Evidencia obtenida de estudios analíticos bien diseñados de poblaciones o casos y controles con preferencia de más de un centro o grupo de investigación

II-3 Evidencia obtenida de series temporales múltiples con intervención o sin ella.

III: Opiniones de autoridades respetadas, basadas en la experiencia clínica, estudios descriptivos e informes de casos, o informes de comités de expertos.

\section{FUERZA DE LA RECOMENDACIÓN}

A: Existen evidencias sólidas para apoyar la inclusión de la actividad

B: Existen suficientes evidencias para apoyar la inclusión de la actividad

C: No existen evidencias suficientes para recomendar o rechazar la inclusión de la actividad, pero es posible hacer recomendaciones apoyándose en otros motivos

D: Existen suficientes evidencias para rechazar la inclusión de la actividad

E: Existen evidencias sólidas para apoyar rechazar la inclusión de la actividad 
Force (USPSTF): Guide to Clinical Preventive Services de 1996 .

Se ha efectuado una revisión sistemática con tablas de evidencia de cada guía y se han seleccionado las actividades más consensuadas o con más respaldo de evidencias de alta calidad.

\section{RESULTADOS}

Los resultados se exponen en la Tabla II.

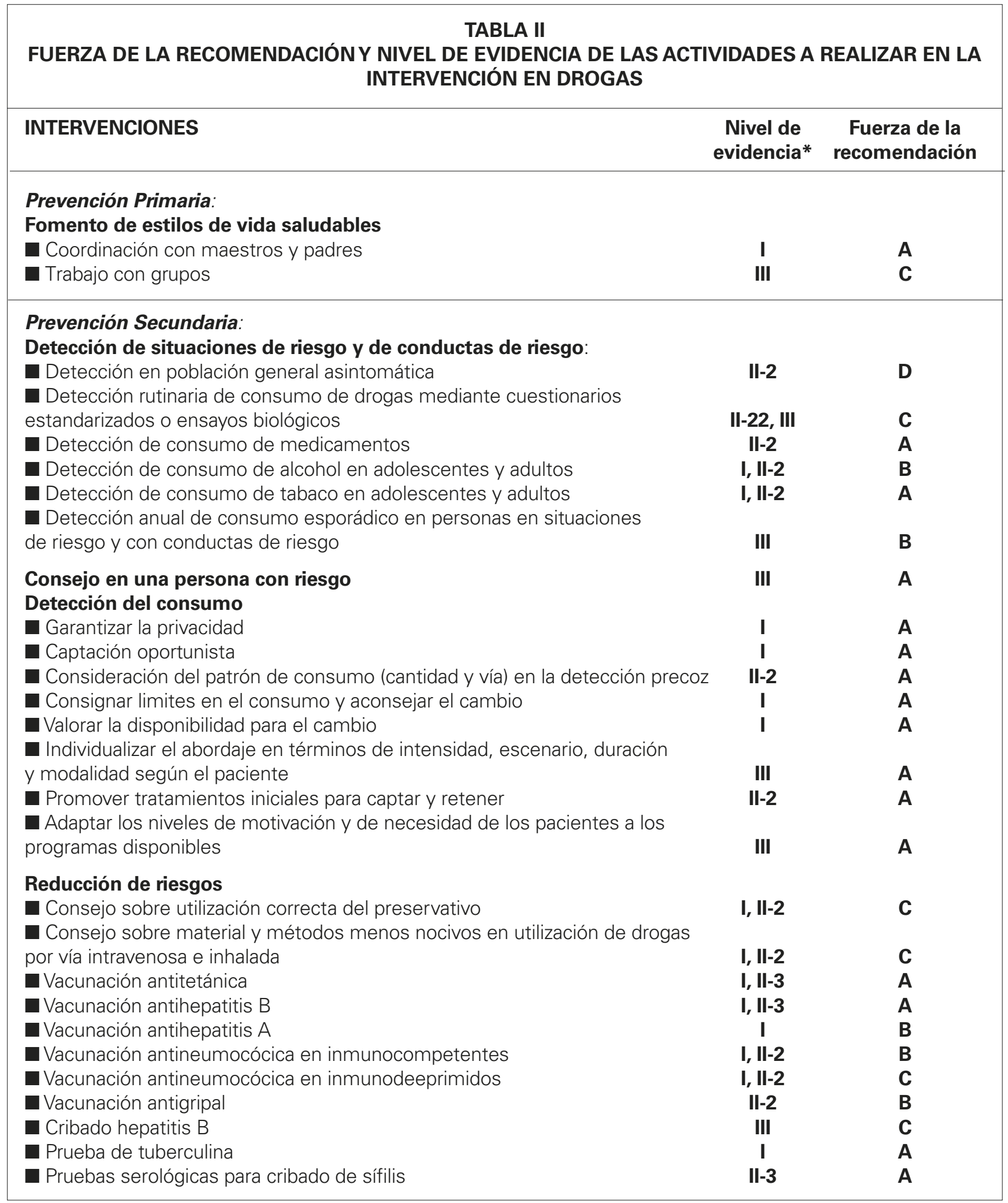


Pruebas serológicas para cribado de gonorrea en mujeres

Pruebas serológicas para cribado de gonorrea en varones

- Pruebas serológicas para cribado de VIH en adolescentes, adultos y mujeres embarazadas de riesgo

- Pruebas serológicas para cribado de clamyidia en mujeres de riesgo elevado

I, II-2, III

- Pruebas serológicas para cribado de clamyidia en varones

II-3, III

B

- Evaluación y control de la tuberculosis

- Cribado de riesgo de suicidio

- Cribado de riesgo elevado de violencia

- Informar a los pacientes de los resultados del despistaje

Considerar la terapia agonista opioide como tratamiento de primera línea para la dependencia opioide

- El mantenimiento con metadona a dosis adecuadas es eficaz en la reducción del uso de opioides

\section{Diagnóstico y tratamiento de las intoxicaciones agudas}

- Evaluación, tratamiento, estabilización y derivación en situación adecuada

\section{Diagnóstico y tratamiento de las enfermedades asociadas a las}

\section{drogodependencias}

- Valoración estandarizada de síntomas coadyuvantes

- Coordinación con el segundo nivel

- Descartar patología dual

- Tratamiento concurrente de los desórdenes psiquiátricos, incluyendo la farmacoterapia

\section{Inicio y seguimiento de algunas desintoxicaciones}

- Captación en Atención Primaria y derivación si preciso

- Fijar criterios para la desintoxicación según niveles

- Establecer los objetivos del tratamiento a través de la negociación

- Revisar los tratamientos previos

- Considerar el uso de valoración estandarizada de lños síntomas de abstinencia

- Facilitar el compromiso con un tratamiento para la deshabituación

I

III

II-1

III

II-2

II-2

\section{Prevención terciaria:}

\section{Rehabilitación (deshabituación)}

- Negociar objetivos específicos con el paciente

- Revisar tratamientos previos

- Comunicación basada en la empatía

- Indicar que la terapia psico-social es efectiva

- Consejo individual y grupal

- Refuerzo motivacional

- Coordinación entre los recursos del sistema y los centros de atención al drogodependiente

II-1
III
I
I
I

\section{Reinserción social}

Técnicas psicológicas y de apoyo social

\section{Control evolutivo}

- Establecer consultas programadas para identificar problemas que incrementan el riesgo de recaída

- Discutir la recaída como un signo para reevaluar el plan de tratamiento

- Modificar los planes de tratamiento basados en las condiciones clínicas y psicosociales del paciente

- Monitorización del uso de sustancias

Cuidados paliativos y de atención a domicilio

$\begin{array}{ll}\text { II } & \text { A } \\ \text { III } & \text { A } \\ \text { III } & \text { B } \\ \text { III } & \text { B } \\ \text { I } & \text { A }\end{array}$

\section{Abordaje familiar}

- Garantizar y explicitar la confidencialidad

I

A

A


- Proporcionar apoyo psicológico de manera planificada

- Coordinación con otros recursos, especialmente en la fase de deshabituación

Abordaje de problemas psicosociales concomitantes

- Terapia conductista en la pareja

\section{Abordaje comunitario}

- Considerar el refuerzo y el abordaje comunitario

- Enfatizar la adherencia a tratamientos de los programas comunitarios

- Complementar desde el sistema el soporte a intervenciones comunitarias y facilitar el desarrollo del soporte comunitario

- Identificar los problemas concurrentes a nivel comunitario para mejorar los resultados

$\begin{array}{cc}\text { I } & \text { A } \\ \text { II-2 } & \text { A } \\ \text { I } & \text { A } \\ \text { I } & \text { A }\end{array}$

A

A

A

A

\section{Coordinación entre niveles}

El abordaje de este problema debe realizarse en todos los niveles del sistema sanitario.

\section{Coordinación intersectorial}

Los servicios sociales de alta calidad han demostrado conseguir mayores niveles de adherencia a la intervención

\section{Formación de los profesionales}

La formación en clínica y en habilidades en terapia cognitivo-conductista para intervenciones breves

\section{Investigación sobre impactos de las intervenciones}

- Mejorar la calidad de las evidencias

- Medición de los impactos de las intervenciones propias

\section{Prevención Primaria: Fomento de estilos de vida}

Es una actividad que se recomienda realizar precozmente desde la infancia y en colaboración clara con los que más influencia tienen en estos momentos que son los educadores (padres y maestros) 2,13. El fomento de estilos de vida saludables en los adolescentes es de especial relevancia, pero sobre todo y fundamentalmente favorecer la comunicación entre padres e hijos. No tiene sentido ocuparse de las drogas sin ocuparse de los adolescentes, sin abordar el conjunto de dificultades y conflictos en los que están muchos de ellos. La prevención no pasa necesariamente por hablar de drogas (aunque alguna vez habrá de hacerse), sino por ayudarle, por ejemplo, a ser miembro de un grupo sin perder su independencia, poner a su alcance ilusiones y experiencias atractivas como las de cualquier droga; pasa por fomentar la independencia e iniciativa. Esta prevención ha de sustentarse en diferentes estructuras: la familia, la escuela, la entidad de tiempo libre o de participación ciudadana. Favorecer la autonomía, el autocontrol, la autoestima, un sistema de valores y la responsabilidad son elementos más importantes en este momento que hablar de entrada de lo malas que son las drogas ${ }^{2,4}$. El consejo al menos una vez al año sobre hábitos de vida saludables en todas las actividades preventivas en consulta y en las grupales, y también en las consultas oportunistas por problemas de salud tiene un nivel de evidencia III $\underline{\underline{C}}^{4,6,8,9,13}$

Mientras que el consejo individual y el trabajo con grupos por profesionales sanitarios es considerado una actividad III $\underline{C}^{4}$, cuando está coordinado y dirigido por los profesionales más competentes en la formación de niños y adolescentes que son los maestros tiene un nivel de Evidencia I $\underline{A}^{13}$.

\section{Prevención Secundaria}

La detección de situaciones de riesgo y de conductas de riesgo así como la detección precoz del consumo son actividades que deben realizarse desde los recursos normalizados de la comunidad y el Equipo de Atención Primaria (EAP) es el que mejor lo puede hacer ${ }^{13}$.

Detección de situaciones de riesgo y de conductas de riesgo:

Los niños y adolescentes con fuertes presiones sociales, patrones familiares anómalos, déficits en la socialización... se hallan en situación de riesgo y determinadas conductas deben hacernos pensar en un consumo de drogas: cambios bruscos de actitud y de humor, ausencias a clase o al trabajo, empeoramiento significativo de la relación con la familia o con los amigos, resistencia a cumplir las normas de 
casa, incumplimiento de horario, brotes de malhumor repentinos e inusuales, pérdida de la comunicación, aumento de petición de dinero a padres o amigos, robos en casa, en la escuela... ${ }^{2}$ Mientras que la detección en población general asintomática tiene un nivel de evidencia II-2 $\underline{D}^{5,8,9,10,12,13}$ y la detección rutinaria de consumo de drogas mediante cuestionarios estandarizados o ensayos biológicos tiene un nivel de evidencia II-2, III y una fuerza de recomendación $\underline{C}_{1}^{4,5,9,10,12,13}$ la detección de consumo de medicamentos (II-2 $\underline{A}$ ), la detección de consumo de alcohol en adolescentes $y$ adultos (I, II-2 B $)$ y de tabaco (I, II-2 A) tienen mayor fuerza para su recomendación de forma rutinaria ${ }^{4-13}$. Sin embargo el despistaje anual de consumo esporádico en personas en situaciones de riesgo y con conductas de riesgo tienen un nivel de evidencia III con una fuerza de recomendación $\underline{B}^{5,8,9,13}$. El consejo en una persona con riesgo tiene un nivel de evidencia III y una fuerza de recomendación $\underline{A}^{13}$.

\section{Detección del consumo:}

Ante situaciones y conductas de riesgo se debe interrogar directamente con adecuado manejo de las habilidades de comunicación y garantizando la privacidad $(\underline{I A})^{13}$. Con los adolescentes la captación debe ser oportunista $(I \underline{A})^{4,8,13}$. Una vez confirmado el consumo el médico de familia debe valorar el patrón de consumo actual (en el último mes), la delimitación del uso de las drogas (uso problemático, consumo perjudicial, dependencia...) las circunstancias familiares y sociales (genograma), la historia breve, los hábitos de riesgo, los antecedentes personales, exploración y exploraciones complementarias ${ }^{2,14}$. Se relacionan a continuación las actividades que, en este momento, se consideran recomendables 2,4,13:

- La consideración del patrón de consumo (cantidad y vía) en la detección precoz II-2 $\underline{A}$.

- Consignar limites en el consumo y aconsejar el cambio (IA)

- Valorar la disponibilidad para el cambio (IA)

- Individualizar el abordaje en términos de intensidad, escenario, duración y modalidad según el paciente (III $\underline{A})$

- Promover tratamientos iniciales para captar y retener (II-2 $\underline{A})$

- Adaptar los niveles de motivación y de necesidad de los pacientes a los programas disponibles (IIIA)

Reducción de riesgos:

Las posiciones maximalistas sólo han servido para perder la relación con un importante grupo de drogodependientes ${ }^{2}$. El objetivo es mantener al drogodependiente en niveles de salud aceptables y mejorar su calidad de vida ${ }^{1,2}$ :
- Consejo sobre medidas higiénicas que eviten los riesgos asociados a la adquisición y transmisión de enfermedades: utilización correcta de preservativo (I, II-2 C), material y métodos menos nocivos en utilización de drogas por vía intravenosa e inhalada (I, II-2 $\underline{C})^{13}$.

- Vacunaciones: antitetánica (I, II-3 $\underline{A})^{4,7,8,9,13}$, antihepatitis $B(I,||-3 \underline{A})^{4,7,8,9,13}$ y $A(\mid \underline{B})^{4,7,8,9,13}$, antineumocócica (I, II-2 $\underline{B}$-en inmunocompetentes- y I, II-2 $\underline{C}$-en inmunodeprimidos) 4,7,8,9,13, antigripal (II2 B $)^{4,7,8,9,13}$

- Cribado de Hepatitis B (III $\underline{C})^{4,7,8,9,13}$; prueba de la tuberculina (I $\underline{A})^{4,7,8,9,13}$; pruebas serológicas para cribado de sífilis (II-3 $\underline{A})^{4,7,8,9,13}$, gonorrea (II-2, III, $\underline{B}$ en mujeres II-3 y III $\underline{C}$ en varones) 4,7,8,9,13; cribado de $\mathrm{VIH}$ en adolescentes, adultos y mujeres embarazadas de riesgo (I, II-2 $\underline{A})^{4,7,8,9,13}$, Cribado de clamidia (I, II-2 y III $\underline{B}$ en mujeres de riesgo elevado y II3 y III $\underline{C}$ en varones) ${ }^{4,7,8,9,13}$.

- Evaluación y control de la tuberculosis (IA) 4,7,8,9,13

- Cribado de riesgo de suicidio (II-2 $\underline{A})^{13}$

- Cribado de riesgo elevado de violencia (III $\underline{A})^{13}$

- Informar a los pacientes de los resultados del despistaje (IIA) ${ }^{13}$

Diagnóstico y tratamiento de las intoxicaciones agudas

En algunas ocasiones el médico de familia debe atender situaciones de urgencia por intoxicaciones agudas ya sea por opiáceos y por psicoestimulantes. Una vez realizada la evaluación (descartar hipoglucemia, rabdomiolisis, traumatismo craneal, edema agudo de pulmón) es necesario corregir la hipoxia y recoger muestras de orina y sangre para realización de pruebas complementarias, instaurar tratamiento si es por opiáceos (Naloxona) y una vez estabilizado el paciente, este debe ser derivado al hospital en situación adecuada $(I I \mid \underline{A})^{2,13}$.

Diagnóstico y tratamiento de las enfermedades asociadas a las drogodependencias:

Los problemas orgánicos de los adictos a drogas se relacionan básicamente con las infecciones por lo que hay que conocer la metodología de la drogadicción, los hábitos sociales y sexuales, repercusión sobre el organismo (patología dental y periodontal, la malnutrición, disminución del reflejo de la tos, traumatismos y erosiones cutáneas...que favorecen las infecciones), fuentes de infección (piel, material de inyección y disolventes y sangre u otros fluidos orgánicos), los factores de riesgo. Se debe tener en cuenta que las infecciones más importantes (por frecuencia o gravedad) son las infecciones de partes blandas, artritis y osteomielitis, infecciones endovasculares, pleuropulmonares, del sistema nervioso central, candidiasis, 
de transmisión sexual, hepatitis B y C, VIH / SIDA². Considerar la valoración estandarizada de síntomas coadyuvantes se considera una intervención II-2 $\underline{A}^{13}$. Algunas de estas patologías requerirán la intervención del segundo nivel, con el que se deberá trabajar de forma coordinada $(I \underline{A})^{13}$.

Es conveniente, asimismo, descartar en estos casos la patología dual, es decir, la coexistencia de una drogodependencia y otro trastorno mental (I $\underline{A})^{13}$ y derivar para instaurar el tratamiento concurrente de desórdenes psiquiátricos incluyendo farmacoterapia $(\underline{I} \underline{A})^{13}$.

Inicio y seguimiento de algunas desintoxicaciones:

No es infrecuente que alguno de nuestros pacientes drogodependiente acuda a nuestra consulta solicitando un tratamiento de desintoxicación y los médicos de familia debemos saber cuando está indicada nuestra intervención o cuando es más adecuado derivar a un centro de atención al drogodependiente $(I \underline{A})^{13}$.

Son de elección para su abordaje en Atención Primaria: Usuarios jóvenes, pacientes que no han realizado tratamientos previos, pacientes que habiendo realizado tratamientos previos, han tenido períodos de abstinencia considerables y se encuentran en situación de recaída, pacientes con los que previamente se tenía una buena relación que permite el control de los riesgos de manipulación, usuarios con buena cobertura familiar y social, con buena motivación y que no existan ganancias secundarias con la desintoxicación, usuarios sin patología dual ni orgánica que dificulte el manejo de la desintoxicación ${ }^{2}$. Fijar indicaciones para la desintoxicación según niveles de atención se considera III $\underline{A}^{13}$.

Esta guía establece asimismo un protocolo de actuación para los médicos de familia ante un proceso de desintoxicación. Establecer los objetivos del tratamiento a través de la negociación ( II-1 $\underline{A}$ ) y revisar los tratamientos previos (III B $)$; considerar el uso de valoración estandarizada de los síntomas de abstinencia (II-2 A); Durante la desintoxicación, facilitar el compromiso con un tratamiento para la deshabituación (II-2 $\underline{A})^{2,13}$.

\section{Prevención Terciaria}

\section{Rehabilitación y reinserción social}

La segunda fase en todo tratamiento de rehabilitación, tras la desintoxicación, es la deshabituación. Una vez superada la fase en la que el objetivo es tratar de superar la sintomatología asociada al síndrome de abstinencia, se entra en un proceso de mantenimiento de la abstinencia dónde las técnicas psicológicas y de apoyo social que posibiliten una efectiva reinserción en la sociedad son la clave, con un nivel de evidencia I y una fuerza de recomendación $\underline{A}^{13}$. En este sentido se revisan las actividades a realizar a este nivel:
Negociar objetivos específicos con el paciente (II-1 A), revisar tratamientos previos y esfuerzos realizados por el paciente para el cambio (III $\underline{B}$ ), usar estilo de terapia basada en la empatía y el no juzgar (frente a la confrontación) (IA $)$, indicar al paciente que la terapia psicosocial es efectiva (IA $)$, respetar las preferencias del paciente para el inicio del abordaje psicosocial (IA), considerar el consejo individual y en grupo (IA), considerar el refuerzo motivacional (IA). En este momento la actuación principal pivotara sobre los centros de atención al drogodependiente y el elemento clave es la coordinación $(\mid \underline{A})^{1,2,13}$.

\section{Control evolutivo}

Una vez realizadas las intervenciones es conveniente mantener lo más posible el contacto y la accesibilidad, pautando visitas programadas de seguimiento por un lado y por otro favorecer nuestra accesibilidad ante algunas eventualidades ${ }^{2}$.

Es importante establecer consultas programadas con el objetivo de identificar problemas que incrementan el riesgo de recaída (II $\underline{A})$, discutir la recaída como un signo para reevaluar el plan de tratamiento (III $\underline{A}$ ) y modificar los planes de tratamientos basados en las condiciones clínicas y psicosociales del paciente $(\mathrm{III} \underline{B})$. La monitorización futura de uso de sustancias tiene un nivel de evidencia III y una fuerza de recomendación $\underline{B}^{13}$.

\section{Cuidados Paliativos y de Atención a domicilio}

En algunas ocasiones nos encontramos con pacientes drogodependientes en fase terminal. Y su abordaje es igual al de cualquier otro paciente en esta situación, debiéndose desarrollar desde Atención Primaria en la medida en la que el paciente esté en su domicilio, con el apoyo si es preciso de otros recursos (IA) ${ }^{1,2,3,13,14}$. Deberán atenderse los síntomas: dolor, digestivos, respiratorios, metabólicos, psiquiátricos, neurológicos, urinarios, dermatológicos, las urgencias que puedan ir apareciendo. Asimismo se atenderá la fase agónica, los aspectos psicosociales y la familia en esta fase.

\section{Abordaje Familiar}

El abordaje familiar es una tarea si no exclusiva, muy vinculada a las funciones del médico de familia y estos temas constituyen una prioridad de intervención familiar que debe desarrollarse a dos niveles:

- Orientación familiar anticipada y ciclo vital

- Abordaje familiar especifico de familias con pacientes drogodependientes 
La orientación familiar anticipada cobra especial interés por su capacidad para prevenir y por lo tanto es importante su realización en familias con adolescentes y más si se detectan riesgos en dicha familia ${ }^{14}$.

Es conveniente siempre garantizar y explicitar la confidencialidad $(\mid A)^{13}$. Se debe ${ }^{14}$.

- Realizar un genograma y analizar el sistema relacional: uso de libertad, sentido de la responsabilidad, grado de dependencia del adolescente y relaciones con los padres

- Asesorar a los padres sobre normas de conducta y mantenimiento de la comunicación

- Tratar sobre la pubertad y la sexualidad

- Detectar conductas de riesgo: tabaco, medicamentos psicotropos, alcohol, drogas, delincuencia, fracaso escolar, embarazo, accidentes...

- Atención a otros problemas de salud: riesgo de suicidio, trastornos del comportamiento alimentario, anorexia y bulimia, discapacidad.

- Red social

En relación al abordaje familiar especifico de familias con pacientes drogodependientes ${ }^{13,14}$ : se deberá tener en cuenta el inicio, el pronóstico, así como los recursos familiares, el momento de presentación, el familiar afectado, las creencias y comportamientos y la evolución. Es necesario proporcionar apoyo psicológico no sólo al principio sino a lo largo de todo el proceso, de una manera planificada y dosificada (IA $)$. En estos casos puede ser necesaria la coordinación con otros recursos, especialmente en la fase de deshabituación (IA). El abordaje de problemas psicosociales concomitantes se considera IA. La terapia conductista en la pareja es considerada nivel I $\underline{A}$.

\section{Abordaje Comunitario}

La oferta de servicios de atención a las conductas adictivas debe estar en principio en el paquete mínimo de oferta de todos los centros de salud, si bien es cierto que aquellos centros de salud en los que este problema sea prioritario por su extensión y gravedad debe realizarse un programa de salud en el que no sólo se contemple el aspecto de intervención sino también el de participación comunitaria, movilizando todos los recursos socio-sanitarios a nuestro alcance ${ }^{2}$. Considerar el refuerzo y el abordaje comunitario es considerado $\underline{A}^{13}$; Enfatizar la adherencia a tratamientos de los programas comunitarios: $\mid \underline{A}$; Complementar desde el sistema el soporte a intervenciones comunitarias o /y facilitar el desarrollo del soporte comunitario: II-2펴 ; identificar los problemas concurrentes a nivel comunitario para mejorar los resultados es $I \underline{A}^{13}$.

\section{Coordinación entre niveles}

Quizá uno de los grandes problemas que ha acompañado al abordaje de las drogodependencias ha sido la falta de "visualización" y por lo tanto de coordinación entre los diferentes niveles de atención. Establecer y conocer la red de recursos, tanto de servicios de información como de centros de atención al drogodependiente, centros de día, programas de metadona, unidades de desintoxicación hospitalaria y comunidades terapéuticas y favorecer su coordinación estable y fluida con los recursos "normalizados" del sistema sanitario es una premisa ineludible. Los médicos de familia debemos conocer los recursos a nuestro alcance para poder orientar a nuestros pacientes sobre estos y sobre las posibilidades terapéuticas ${ }^{2}$.

Esto implica también la coordinación con el segundo nivel sobre todo para el control y seguimiento de la patología somática asociada a las drogas. Hay múltiples estudios que refuerzan que el abordaje a este problema debe realizarse en todos los niveles del sistema sanitario $(\mid \underline{A})^{13}$.

\section{Coordinación intersectorial}

Si uno de los males en el abordaje de este problema ha sido la falta de relación entre los niveles asistenciales también lo ha sido y desde luego con no menor impacto la ausencia de la misma entre sectores implicados. Es de absoluta necesidad que exista coordinación del sistema sanitario con la comunidad y sus recursos, con el sector educativo y con el sector de recursos sociales, estableciendo mecanismos estructurales que "fuercen esta coordinación" 2. Existen pocos temas en los que sea tan importante y necesario el abordaje integral y multidisciplinar como lo representa este tema. Los servicios sociales de alta calidad han demostrado conseguir mayores niveles de adherencia a la intervención (IA $)^{13}$.

\section{Formación de los profesionales}

Cuando se analiza la actitud de muchos de nosotros frente al abordaje de este problema, se observan dos causas importantes para "el escaso interés" mostrado hacia éste: una vinculada a la inexistente coordinación entre Atención Primaria y la red especifica, al menos de forma formal, y otra a problemas estructurales tan importantes como la falta de tiempo y al sentimiento de muchos profesionales sobre su insuficiente nivel de formación en estos temas ${ }^{2}$.

Con el fin de mejorar la formación en estos temas, la atención a las conductas adictivas ha sido incluida como área formativa en el nuevo programa de la espe- 
cialidad de Medicina Familiar y Comunitaria aprobado por la Comisión Nacional de la Especialidad el 20-XI$2002^{3}$.

La formación en la clínica y la formación en habilidades de terapia cognitivo-conductista para intervenciones breves son intervenciones $\mid A^{13}$.

\section{DISCUSIÓN}

De entre todas las guías revisadas, se evidencia cierto grado de heterogeneidad entre ellas, fundamentalmente por la amplitud de las actividades analizadas. Todas ellas contemplan y analizan actividades de prevención primaria y secundaria. Mientras la AMA $^{10}$ y la AAFP ${ }^{12}$ aconsejan a los médicos incluir una historia en detalle respecto a las toxicomanías como parte de un examen completo de salud para todos los pacientes, la USPSTF ${ }^{8}$ considera la inclusión de preguntas relacionadas con consumo de drogas como una recomendación $\mathrm{C}$, pudiendo recomendarse por otros motivos; la $\mathrm{VHA}^{13}$, sin embargo, considera que la detección de pacientes asintomáticos es una recomendación $D$ (II-2). Estas dos últimas consideran la detección rutinaria de consumo de drogas mediante cuestionarios estandarizados o ensayos biológicos como una actividad III C. Todas las organizaciones recomiendan los exámenes de orina cuando hay sospecha razonable de toxicomanía, pero ninguno de estos grupos recomienda pruebas para la detección rutinaria de drogas en ausencia de indicaciones clínicas ${ }^{4-13 .}$

Existe también un importante nivel de acuerdo sobre la captación oportunista, garantizando la privacidad en los adolescentes y sobre la consideración del patrón de consumo, la valoración de la disponibilidad para el cambio, el consejo para que éste se produzca y los límites para el consumo y la adaptación de los niveles de motivación y de necesidad de los pacientes para los programas disponibles. También existe un consenso importante sobre las actividades de reducción de riesgos reflejada en la tabla || 4-13.

De todas las guías revisadas, hay que destacar la guía de práctica clínica de la $\mathrm{VHA}^{13}$, por su amplia consideración de las actividades a realizar, y por su consideración de las actividades propias de atención primaria, de otros niveles y sectores y de la importancia de la coordinación. Esta guía incluye 207 referencias y basa sus conclusiones en ensayos clínicos del registro de Cochrane, basando preferentemente sus conclusiones en meta-análisis, ensayos clínicos o estudios caso-control. Incluye, como las guías anteriores, no sólo actividades de prevención primara y secundaria de detección de situaciones de riesgo, del consumo, reducción de riesgos y control evolutivo, sino también los cuidados paliativos y la atención a domicilio, el abordaje familiar y comunitario, la coordinación entre niveles e intersectorial y la formación de los profesionales.

Las evidencias científicas de alta calidad demuestran que el abordaje en las conductas adictivas debe realizarse en todo el sistema sanitario, siendo la coordinación entre los niveles sanitarios, entre los sectores y entre los recursos comunitarios, así como la formación de los profesionales, la clave del éxito.

Este tema afortunadamente tiene ya muchas respuestas basadas en la evidencia, pero es cierto que algunas se basan en evidencias de nivel III. Mejorar la calidad de las evidencias, junto con la medición de impactos propios de las intervenciones a nivel de la comunidad son áreas de interés para seguir investigando.

Los médicos de familia somos los únicos especialistas que no podemos decir "esto no es mío" y, por lo tanto, este tema tan trascendente no puede ser eludido. No podemos ni debemos mantenernos al margen y lo debemos hacer bien y de forma coordinada. Las carteras de servicios de Atención Primaria, paulatinamente, incorporan la atención al tabaquismo, al alcoholismo y a las drogodependencias y nuestra actitud debe ser no sólo de aceptación sino proactiva y amplia y a todos los niveles: atención, formación, investigación, planificación, organización, gestión y coordinación.

\section{REFERENCIAS}

1. semFYC. Guía de actuación en Atención Primaria. $2^{a}$ Edición. semFYC. Barcelona 2002

2. Navarro Cañadas, C. et al. Guía de Manejo de las Drogodependencias en Atención Primaria. Sociedad Castellana y Leonesa de Medicina de Familia y Comunitaria. Valladolid. 2002.

3. Comisión Nacional de la Especialidad de Medicina Familiar y Comunitaria. Programa de la Especialidad de Medicina Familiar y Comunitaria (aprobado el 20-XI2002) URL locate www.semfyc.es.

4. PAPPS. Actualización 2003. Aten Primaria. 32 (supl 2). 2003: 1-158.

5. US Preventive Services Task Force. Adicción a Drogas. Guía de Medicina Clínica Preventiva. Barcelona. Medical Trends SL. 2002: vol 3 : 465-76

6. US Preventive Services Task Force. Consejos preventivos para el paciente. Guía de Medicina Clínica Preventiva. Barcelona. Medical Trends SL. 2002: vol 3 : 476-593.

7. US Preventive Services Task Force. Enfermedades infecciosas. Guía de Medicina Clínica Preventiva. Barcelona. Medical Trends SL. 2002: vol 2 : 212-289. 
8. US Preventive Services Task Force. Guide to Clinical Preventive Services. Second edition. Alexandria. Virginia. International Medical Publishing. 1996.

9. Canadian Task Force on the periodic health examination. The periodic health examination. Can Med Assoc J 1979; 121: 1193-254.

10. American Medical Association. Drug abuse in the United States: a policy report. Report of the Board of Trustees. Chicago. American Medical Association, 1988.

11. American Medical Association. Guidelines for adolescent preventive services (GAPS): recommendations and rationale. Chicago. American Medical Association, 1994.

12. American Academy of Family Physicians. Age charts for periodic health examination. Kansas city, MO: American Academy of Family Physicians, 1994.

13. National Guidelines Clearinghouse. VHA/DoD Clinical practice guideline for the management of substance use disorders. Veterans Health Administration/Department of Veterans Affairs/department of Defense. 2002. sep. URL locate www.guidelines.gov/index.asp.

14. Sánchez García JI. Atención a la Familia. SemFYC. SEMERGEN. Barcelona. 2000. 